\title{
Entrenamiento y confiabilidad entre observadores en el análisis delfútbol para ciegos Reliability and inter-coders training in the analysis of football for blind persons
} José Martín Gamonales Puerto ${ }^{1}$, Jesús Muñoz Jiménez ${ }^{1,2}$, Kiko León Guzmán ${ }^{1,2}$ Sergio José Ibáñez Godoy ${ }^{1}$ ${ }^{1}$ Universidad de Autónoma de Chile (Chile), ${ }^{2}$ Universidad de Extremadura (España)

\begin{abstract}
Resumen. El objetivo de este trabajo es describir el proceso de entrenamiento y evaluación de la fiabilidad inter-observador de los codificadores participantes en un estudio sobre el análisis en fútbol a 5 para personas ciegas. Se trata de un estudio instrumental donde cinco observadores seleccionados codificaron las distintas acciones técnicas desarrolladas durante los lanzamientos a portería. Para ello se empleó un proceso de entrenamiento de codificadores con el fin de asegurar una adecuada fiabilidad de los datos, dividido en cuatro etapas: etapa preparatoria; etapa de selección de los codificadores; etapa de formación de los observadores; y etapa de confiabilidad. Durante la etapa formación de los codificadores, la fiabilidad se calculó a través de la prueba Multirather Kappa Free. Los resultados del proceso de entrenamiento muestran una mejora en cada variable y total de la concordancia entre los codificadores. Los datos obtenidos por los codificadores muestran niveles sustanciales de validez y fiabilidad, y por tanto pueden ser aplicados en la investigación sobre el análisis observacional del fútbol a 5 para personas ciegas.
\end{abstract}

Palabras claves: fútbol a 5, ciegos, metodología observacional, confiabilidad, observadores.

Abstract. The aim of this work is to describe the process of coders training and reliability evaluation in the analysis of football 5-a-side for blind persons. This research is instrumental, with five selected observers codifying different technical actions during shots on goal. A coders training process was developed in order to ensure the adequate reliability of data, and divided into four stages: preparatory stage; coders' selection stage; coders' formation stage; and reliability stage. During the training stage of the encoders, reliability was calculated through Multirather Kappa Free tests. The results of the training process show an improvement of the agreement between coders in each variable and overall. The data obtained by coders show substantial levels of validity and reliability, and therefore can be applied in research focusing on observational analysis of 5-a-side football for blind persons.

Key words: football 5-a-side, blind, observational methodology, reliability, observers.

\section{Introducción}

El Fútbol a 5 para personas ciegas (en adelante Fa5) es un deporte de cooperación oposición (Hernández, 2005) que se juega en un terreno descubierto para permitir una acústica óptima para los deportista (IBSA, 2017). Se ha convertido en uno de los deportes más populares dentro del colectivo de personas ciegas o con discapacidad visual en todo el mundo (Gamonales, 2017). Sigue las mismas reglas de la Federación Internacional deAsociaciones de Fútbol (FIFA), con algunas adaptaciones específicas que permiten a los deportistas ciegos y con discapacidad visual jugar (Morato, Bilzon \& Duarte, 2013), atendiendo a sus características y capacidades particulares (Matsui, 2017). Los estudios relacionados con el $\mathrm{Fa} 5$ son de tipo divulgativo y no están relacionados con el análisis del rendimiento deportivo (Gamonales, 2016).

El análisis del rendimiento deportivo es una de las líneas de investigación más relevantes de las Ciencias del Entrenamiento. Son estudios emergentes, que recientemente están ganado popularidad entre los investigadores y profesionales del deporte (Drust, 2010). Este análisis ha evolucionada desde el primer momento con modelos estadísticos descriptivos que permitían caracterizar el perfil de un jugador, equipo o competición pero limitado respecto a su contextualización (Sampaio, Ibáñez \& Lorenzo, 2013). Por tanto, la existencia de una alta complejidad eimprevisibilidad en deporte exige que la Metodología Observacional y de medida sean necesarios para la mejora del conocimiento sobre el rendimiento deportivo (Gómez-Ruano, 2017). El objetivo del análisis del rendimiento deportivo es progresar en el conocimiento del contexto de juego con vistas a mejorar los resultados fututos (McGarry, 2009) durante el entrenamiento o la competición. En el análisis del rendimiento deportivo se incluyen todas las investigaciones que analizan la competición o el entrenamiento (Hughes \& Bartlett, 2002). Es una disciplina que ha ido creciendo y desarrollándose tanto en el número como en la profundidad y rigurosidad de sus investigaciones, gracias al desarrollo de aplicaciones informáticas para el análisis del alto rendimiento (Nevill, Atkinson \& Hughes, 2008), así como a la aplicación de técnicas estadísticas que validan el método utilizado en este tipo de investigaciones.

Por tanto, el análisis de la competición tienen una gran importancia

Fecha recepción: 27-02-17. Fecha de aceptación: 22-11-17

José Martín Gamonales Puerto

josemartingamonales@gmail.com para los investigadores y entrenadores pues ambos están interesados en percibir el tipo de acciones que se asocian a la eficacia de los equipo (Caro \& Caro-Muñoz, 2016). Por ello, los investigadores utilizan diferentes metodologías en sus estudios, así como instrumentos y/o herramientas para la recogida de datos. En la última década se ha producido un incremento relevante en el interés por la utilización de la metodología observacional en el ámbito del deportes (Anguera \& Hernández-Mendo, 2013) y ha adquirido identidad propia. La metodología observacional aplicada en numerosos trabajos para el análisis de la acción del juego en el deportetiene una validez científica verificada(Anguera, 1991; Anguera \& Hernández-Mendo, 2014; Anguera \& Hernández-Mendo, 2015). Es un procedimiento flexible y riguroso en el estudio del juego (Anguera \& Hernández-Mendo, 2013) y posibilidad la elaboración de procedimientos o instrumentos (Sarmento, Anguera, Campaniço \& Leitão, 2010).

Dentro de la metodología observacional, Medina \& Delgado (1999) proponen un modelo de formación de observadores que asegura la fiabilidad en la recogida de datos (Anguera, Blanco, Losada \& SánchezAlgarra, 1999; Losada \& Manolov, 2015). Es una herramienta útil para diferentes contextos y líneas de investigación, generando trabajos de gran calidad que permiten que este área avance con base en su metodología (Drust, 2010). En el ámbito deportivo hay diferentes formas de estimar la fiabilidad, validez y precisión (Anguera, 1991). La base del análisis notacional es la observación, esto implica que los registros de las acciones pasan obligatoriamente por la intervención de los codificadores. Las ciencias de la actividad física y el deporte tiene a su alcance una de las metodologías más relevantes para llevar a la práctica estudios sencillos o investigaciones degran magnitud(Castellano \& Hernández-Mendo, 2015).

En la literatura científica, hay trabajos relacionados con la metodología observacional que utilizan un proceso de formación de los codificadores e incluso detallan las fases seguidas (Tabla 1). La mayoría de los trabajos de investigación para estimar la fiabilidad de los observadores, casi siempre aplican la corrección por efecto del azar con el índice de Kappa de Cohen (Fleiss, Levin \& Paik, 2013). Para los criterios cualitativos se utiliza el coeficiente de Kappa (Casal et al., 2015; Usabiaga et al., 2013; Santos et al., 2014) y para los criterios cuantitativos, las correlaciones de Pearson (Arias et al., 2009). Este método es una forma económica de proporcionar información cualitativa y cuantitativa sobre los jugadores y entrenadores, y además, puede ayudar a proporcionar información relacionada con los aspectos técnicos y tácticos del deporte objeto de estudio (Hughes \& Bartlett, 2002). 
Tabla 1.

\begin{tabular}{|c|c|c|c|c|c|}
\hline Autor / es y año & Deporte o ámbito & $\begin{array}{l}\text { Describe } \\
\text { proceso }\end{array}$ & Totalidad proceso & Form. Teórica & $\begin{array}{c}\mathrm{N}^{\circ} \\
\text { codific. }\end{array}$ \\
\hline Moreno et al., (2002) & Voleibol & Sí & No define & 3 sesiones (4h $30 \mathrm{~min})$ & 10 \\
\hline Nadeau, Richard \& Godbout (2008) & Hockey & No & No define & No define & 2 \\
\hline Alonso \& Argudo (2008) & Frontenis Olímpico & No & Cuatro semanas & No define & 4 \\
\hline Taylor, Mellalieu, James \& Shearer (2009) & Fútbol & No & No define & 10 horas & 2 \\
\hline Ibáñez, García, Feu, Parejo, \& Cañadas (2009). & Baloncesto & No & No define & No define & No define \\
\hline Lago (2009) & Fútbol & No & No define & 10 horas & 2 \\
\hline Arias, Argudo \& Alonso (2009) & Baloncesto & Sí & Dos semanas & 6 sesiones $(21 \mathrm{~h})$ & 4 \\
\hline Hernández-Mendo, Montoro, Reina \& Fernández (2012) & Voleibol & No & No define & No define & 6 \\
\hline Usabiaga, Castellano, Blanco \& Casamichana (2013) & Pelota vasca & No & No define & $30 \mathrm{~h}$ & 8 pares \\
\hline Sousa, Prudente, Sequeira \& Hernández-Mendo (2013) & Andebol & Sí & No define & No define & 11 \\
\hline Blanco, Ibáñez, Antúnez \& Hernández-Mendo (2015) & Balonmano & No & 12 sesiones & No define & 2 \\
\hline Villarejo, Ortega, Gómez \& Palao (2014). & Rugby & Sí & 12 Sesiones & 3 sesiones (6h) & 3 \\
\hline Santos, Sarmento, Alves \& Campanico (2014) & Waterpolo & Sólo fases & No define & No define & 3 \\
\hline Morillo \& Hernández-Mendo (2015) & Balonmano playa & No & No define & No define & No define \\
\hline Casal, Losada \& Ardá (2015) & Fútbol & No & No define & No define & 4 \\
\hline Catalán-Eslava \& González- Víllora (2015) & Squash & Sí & No Define & 5 sesiones & 6 \\
\hline Serra-Olivares \& García-López (2016) & Fútbol & Sí & No define & No define & 7 \\
\hline García-Martín, Antúnez \& Ibañez (2016) & Formación jugadores & $\mathrm{Si}$ & No define & No define & 11 \\
\hline $\begin{array}{l}\text { Robles-Rodríguez, Robles-Rodríguez, Giménez Fuentes- } \\
\text { Guerra, \& Abad } 2016\end{array}$ & Judokas & Sí & No define & No define & 10 \\
\hline Morato, Da Cunha, Gamero, Magalhães \& Almeida (2016) & Goalball & No & No define & 2 sesiones & 2 \\
\hline
\end{tabular}

cinco criterios de inclusión establecidos en el estudio. Los criterios seleccionados para la inclusión como codificadores fueron:

Primer criterio. Mostar interés en el estudio.

Segundo criterio. Poseer una titulación universitaria relacionada con la Actividad Física y el Deporte (Licenciatura / Graduado en Ciencias del Deporte) o la Educación Física (Diplomado / Graduado en Educación, especialidadEducación Física).

Tercer criterio. Tener formación específicas relacionadas con la actividad física para personas con discapacidad. Cuarto criterio. Tener publicaciones de carácter didáctico y/o científico rela-

La metodología observacional es utilizada de forma exitosa en un amplio espectro de estudios (Anguera \& Hernández-Mendo, 2015) que abarcan las diferentes modalidades deportivas. La observación como método de investigación se encuentra en continua evolución gracias a los avances tecnológicos. Además, para que los datos observados puedan convertirse en científicos es necesario que sean fiables y validos. Por tanto, debe seguirse un proceso riguroso (Castellano \& HernándezMendo, 2015).

Tras la revisión realizada, y conociendo las principales propuestas de formación de codificadores (Anguera et al., 1999; Losada \& Manolov, 2014; Medina \& Delgado, 1999), el presente artículo se plantea como una contribución para aumentar el conocimiento relacionado con el objeto de estudio posibilitando mejoras en el trabajo científico. Por ello, el objetivo general de esta investigación es exponer un documento donde se describa el proceso de formación y evaluación de la fiabilidad interobservador de los codificadores participantes en un estudio sobre el análisis del rendimiento deportivo en Fa5 y mejorando los procedimientos descritos en la literatura. Este objetivo general, se operativiza en tres objetivos específicos: i) diseñar y proponer las etapas de un proceso de formación de observadores ii) analizar la validez y fiabilidad de los codificadores y iii) realizar un análisis de confiabilidad del proceso de formación. De esta manera, se podrá estimar el grado de precisión con el que se está midiendo las variables del estudio, pues la confiabilidad indica la consistencia del proceso demedición o de los resultados(Barraza, 2007).

\section{Metodología}

\section{Diseño}

Investigación perteneciente a los estudios instrumentales (Montero \& León, 2007) donde se desarrolla una metodología de entrenamiento de codificadores que asegure la fiabilidad en la recogida de datos.

\section{Participantes}

En el presente estudio participaron cinco observadores. Analizaron distintas acciones básicas durante el lanzamiento a portería en situaciones de juego, y otras para las acciones básicas durante el penalti en Fa5, correspondiente a diferentes Campeonatos organizados por la Federación Internacional de Deportes para Ciegos (IBSA) y Comité Olímpico. Para ello, se tomó como referencia la propuesta de Medina y Delgado (1999) para la formación de codificadores que están involucrados en la definición de las variables y categorías a observar. Por este motivo, se seleccionaron de forma deliberada e intencional (Anguera \& HernándezMendo, 2013). Se buscaron participantes expertos capaces de transmitir conocimientos acerca del objeto de estudio, así como realizar valoraciones al respecto que pueden provocar reflexiones y servir de ayuda al investigador (Escobar \& Cuervo, 2008), y además accesibles.

La muestra de participantes seleccionados para formar parte del proceso de formación deben de cumplir como mínimo con cuatro de los cionadas con la actividad física y las personas con discapacidad. Quinto criterio. Tener al menos dos años de experiencia como técnico deportivo para personas con discapacidad visual.

\section{Variables}

Las variables a observar por los codificadores serán las que constituyen el instrumento IOLF5C (Gamonales et al., In press), cuya finalidad es analizar la eficacia del lanzamiento en fútbol para ciegos. El instrumento se divide en dos partes, una para la evaluación de las acciones básicas durante el lanzamiento a portería en situaciones de juego, y otras para las acciones básicas durante el penalti en Fa5.

\section{Instrumentos}

Para la formación de codificadores y observación de partidos así como para el registro, codificación y tratamiento de los datos se utilizó: una plataforma virtual en entorno Moodle; distintos manuales (presentación de la modalidad deportiva, descripción del estudio y variables); el instrumento IOLF5C; vídeos de las distintas acciones analizar por los observadores; el programa online Multirater Kappa Free; y un servicio de alojamiento de archivos multiplataforma en la nube.

\section{Procedimiento}

La propuesta de Medina \& Delgado (1999), hace referencia a la formación de codificadores que están involucrados en la definición de las variables y categorías a observar. Esto es diferente al rol que los codificadores tienen en el presente estudio, en el que las variables y las categorías han sido definidas previamente y revisadas por un grupo de expertos. Concretamente, los codificadores utilizarán el instrumento IOLF5C (Gamonales et al., In press). Aun así, en ocasiones no se pueden obtener datos fiables. Hay errores en la observación que provienen del propio observador, y se podrían reducir con una buena formación del mismo. Por esta razón el proceso se simplifica, obviando la

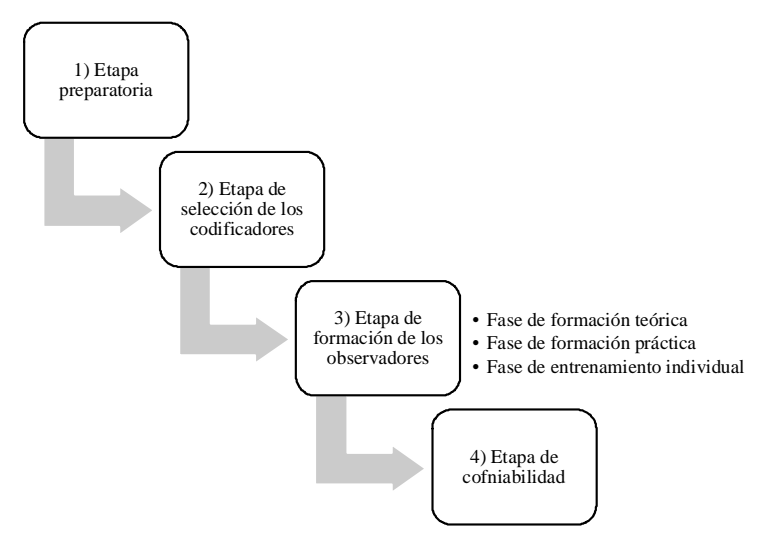

Figura 1. Esquema de las etapas de la investigación. 
división en las dos fases que Medina \& Delgado (1999), Anguera et al. (1999) o Losada \& Manolov (2015) que plantean (preparatoria y entrenamiento) e incluso se tienen en cuenta otras fases con el fin de mejorar de forma constructiva el proceso de formación y existentes en la literatura científica (Arias et al., 2009; Moreno et al., 2002). Para diseñar el proceso de formación de los codificadores, se tuvo en cuenta las fases de entrenamiento y adiestramiento sugeridas por Anguera \& Hernández-Mendo (2013) y se ha realizado de acuerdo a los objetivos específicos del estudio. Este proceso se dividió en cuatro etapas: etapa preparatoria; etapa de selección de los codificadores; etapa de formación de los observadores; y por último, etapa de confiabilidad (Figura $1)$.

1) Etapa preparatoria. Es una etapa relacionada con el tratamiento, organización y gestión de la información. La organización y gestión precisa de la Plataforma Moodle. Es clave para que los observadores seleccionados no tenga dudas sobre el funcionamiento de la misma, así como acerca del propio estudio y proceso de registro. Toda la información que se detalle debe ser sencilla y concisa. Los distintos apartados que constituye el estudios deben quedar claramente identificados en la plataforma. Por cada apartado, hay un foro para que los codificadores puedan preguntar por sus dudas. Los partidos de los distintos campeonatos fueron grabados en MP4. Estos vídeos fueron elegidos de manera aleatoria provenientes de los distintos campeonatos citados anteriormente.

Además, de los foros de consultas y dudas que los codificadores pueden encontrar en la Moodle, se utilizó la aplicación de mensajería WhatsApp para comunicarles cuando se abría cada espacio de la plataforma, informar de los datos de fiabilidad logrados, etc. Se pretendió que los codificadores tuvieran la información más relevante por varios sistemas de comunicación. Todos los datos registrados por los codificadores será guardados en un alojamiento de archivos multiplataforma en la nube. De esta manera, los investigadores se aseguran de no perder la información.

2) Etapa de selección de los codificadores. En esta etapa, se seleccionan los codificadores atendiendo a los criterios de inclusión establecidos en el estudio (Tabla 2).

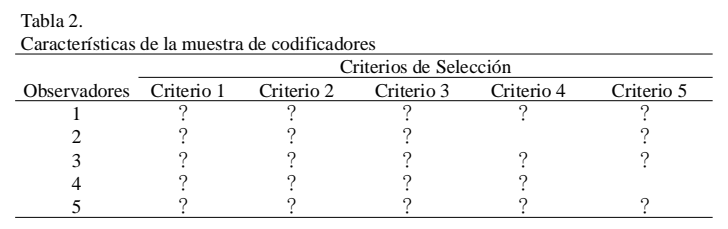

3) Etapa de formación de los observadores. Esta etapa está dividida en tres: fase de formación teórica; fase de formación práctica; y fase de entrenamiento individual.

3.1) Fase de formación teórica. En ella, se pretende que los codificadores adquieran conocimiento, de forma efectiva, en torno a la modalidad objeto de estudio y las variables a registrar. Toda la documentación estaba en formato digital en la plataforma virtual. Esta fase se desarrolla en siete jornadas de forma presencial (Tabla 3). Para el desa- rrollo de la formación se utiliza un ordenador, y se distribuye a los codificadores en torno al mismo.

3.2) Fase de formación práctica. La Plataforma Moodle estaba compuesta por dos apartados para facilitar la compresión a los observadores: fase de formación práctica de observadores (acciones de penalti) y fase de formación práctica (acciones en juego). En cada espacio, se almacenó los vídeos de las acciones de lanzamiento de penalti y lanzamiento en juego en distintas carpetas con 10, 20 y 30 fragmentos de lanzamiento de penalti. Lo mismo sucedió con las acciones de los lanzamiento en juego. El objetivo de la fase es iniciar a los observadores al registro de datos de los vídeos, y comenzar el análisis del nivel de acuerdo inter-codificador, que servirá para evaluar la fiabilidad. Para ello, se utilizó el programa online Multirater Kappa free (Randolph, 2005).

3.3) Fase de entrenamiento individual. De forma progresiva, se evalúa la fiabilidad y se realizan las correcciones y ajustes necesarios para evitar errores en los registros de datos. Por tanto, los codificadores de manera individual deben de observar, registrar los datos de las acciones de juego y penaltis y subir el informe de registro a la plataforma. Además, tras cada análisis de fiabilidad se realizará un informe con los resultados y las recomendaciones de cada una de las variables para darle continuidad al proceso de formación. La estructura de cada uno de los ejercicios que constituyen, tanto la fase práctica como de la fase de entrenamiento es similar, incluyendo todos los mismos recursos salvo los vídeos a utilizar y observar por lo codificadores.

4) Etapa de confiabilidad. Tras la recogida de datos por parte de los codificadores e independientemente de la fiabilidad obtenida en las fases anteriores de la etapa formación de codificadores, se realiza un análisis de confiabilidad. De esta manera, se podrá estimar el grado de precisión con el que se está midiendo las variables del estudio, pues la confiabilidad indica la consistencia del proceso de medición o de los resultados (Barraza, 2007). Por tanto, el objetivo de la etapa fue comprobar que la concordancia consensuada entre los observadores superan los niveles mínimos exigidos (Alonso \& Argudo, 2008).

\section{Análisis estadístico de los datos}

Para evaluar la fiabilidad de los datos recogidos por los codificadores, se llevó a cabo a través del procedimiento estadístico denominado Multirater Kappa free (Randolph, 2005), variante del coeficiente Kappa. Es una herramienta específicamente diseñada para valorar la fiabilidad cuando hay más de dos codificadores que tienen que observar variables que cuentan con más de dos categorías y donde los observadores no están obligados a asignar un número de casos a cada categoría (Brennan \& Prediger, 1981). Randolph (2005) señala que un valor de 0.70 o superior indica un alto grado de acuerdo entre codificadores. Este valor ha sido la medida de referencia adoptada para valorar la fiabilidad intercodificador del presente estudio. Además, se tuvo en cuenta la nomenclatura del nivel relativo de acuerdo entre observadores de Landis \& Koch (1977). Empleadas para facilitar y describir el grado de consenso entre los codificadores durante el periodo de formación. Por último, para el análisis de la etapa de confiabilidad, se calculó el promedio de la fiabilidad de los datos recogidos por los codificadores de forma parcial y total. Esto calculo sirve para comprobar si el proceso de formación de los codificadores que se está evaluando es adecuado.

\begin{tabular}{|c|c|c|c|c|}
\hline Jorn & Denominación & Tiempo & Actividades & $\begin{array}{c}\text { Observaciones / } \\
\text { Material }\end{array}$ \\
\hline 1 & Requisitos previos & $40^{-}$ & $\begin{array}{l}\text { Usuario y contraseña para acceder en la Plataforma Moodle } \\
\text { Grupo de WhatsApp }\end{array}$ & $\begin{array}{l}\text { Cada codificador con su PC. } \\
\text { Cada codificador con su teléfono móvil. }\end{array}$ \\
\hline 2 & $\begin{array}{l}\text { Definición y características del } \\
\text { Fútbol a } 5 \text { para personas ciegas }\end{array}$ & $\begin{array}{ll}20 \\
20\end{array}$ & $\begin{array}{l}\text { Presentación del Fa5 } \\
\text { Presentación de Reglamento del Fa5 }\end{array}$ & $\begin{array}{l}\text { Puesta en común entre los codificadores / Archivo de presentación } \\
\text { Puesta en común entre los codificadores / Archivo reglamento }\end{array}$ \\
\hline 3 & Definición del estudios y variables & $40^{\prime}$ & Presentación del estudio y las variables & Puesta en común entre los codificadores / Archivo descripción del estudio \\
\hline 4 & Variables contextuales. & $40^{-}$ & Presentación variables contextuales y como completar las variables & Puesta en común entre los codificadores / Archivo variables contextuales \\
\hline 5 & $\begin{array}{l}\text { Variables de lanzamiento de penalti } \\
\text { y resultado. }\end{array}$ & $\begin{array}{ll}30 \\
130^{\prime}\end{array}$ & $\begin{array}{l}\text { Presentación variables de penalti y como completar las variables } \\
\text { Presentación variable de resultado de penalti y como completar variables }\end{array}$ & $\begin{array}{l}\text { Puesta en común entre los codificadores / Archivo variables de penalti } \\
\text { Puesta en común entre los codificadores / Archivo variables resultado penalti }\end{array}$ \\
\hline 6 & $\begin{array}{l}\text { Variables de lanzamiento en juego } \\
\text { y resultado }\end{array}$ & $\begin{array}{l}30^{\prime} \\
10^{-}\end{array}$ & $\begin{array}{l}\text { Presentación variables en juego y como completar las variables } \\
\text { Presentación variable de resultado y como completar las variables }\end{array}$ & $\begin{array}{l}\text { Puesta en común entre los codificadores / Archivo variables en juego. } \\
\text { Puesta en común entre los codificadores / Archivo resultado en juego }\end{array}$ \\
\hline 7 & Herramienta de registro y apoyo & $\begin{array}{l}20^{\prime} \\
20^{\prime} \\
20^{-} \\
30^{-}\end{array}$ & $\begin{array}{l}\text { Presentación esquema simplificado variables contextuales } \\
\text { Presentación esquema simplificado variables de penalti y resultado } \\
\text { Presentación esquema simplificado variables en juego y resultado } \\
\text { Ejemplo de ejercicio práctico. }\end{array}$ & $\begin{array}{l}\text { Puesta en común entre los codificadores / Esquema variables contextuales } \\
\text { Puesta en común entre los codificadores / Esquema variables penalti } \\
\text { Puesta en común entre los codificadores / Esquema variables juego } \\
\text { Ejercicio práctico. } \\
\text { Dudas, comentarios y observaciones al ejercicio. }\end{array}$ \\
\hline
\end{tabular}


Tabla 4

Evolución del nivel de acuerdo de los ejercicios prácticos y de entrenamiento de las acciones de lanzamiento a portería.

\begin{tabular}{|c|c|c|c|c|c|c|}
\hline Variable & $\begin{array}{c}10 \text { Vídeos } \\
\text { Lanz. Penalti }\end{array}$ & E.A. & $\begin{array}{c}20 \text { Vídeos } \\
\text { Lanz. Penalti }\end{array}$ & E.A. & $\begin{array}{c}30 \text { Vídeos } \\
\text { Lanz. Penalti }\end{array}$ & \\
\hline VP1. Técnica de orientación & 0.88 & C.P. & 0.94 & C.P. & 0.95 & C \\
\hline VP2. Técnica de lanzamiento & 0.80 & SUS & 0.86 & C.P. & 0.98 & \\
\hline VP3. Zona corporal & 0.96 & C.P. & 1.00 & C.P. & 0.98 & \\
\hline VP4. Tipo de golpeo & 0.80 & SUS & 0.60 & SUS & 0.88 & \\
\hline VP4*. Tipo de golpeo & 0.94 & C.P. & 0.96 & C.P. & 0.96 & \\
\hline \multirow[t]{2}{*}{ VP5. Resultado Penalti } & 0.94 & C.P. & 0.94 & C.P. & 0.98 & \\
\hline & $\begin{array}{l}10 \text { Vídeos } \\
\text { Lanz. Juego }\end{array}$ & E.A. & $\begin{array}{l}20 \text { Vídeos } \\
\text { Lanz. Juego }\end{array}$ & E.A. & $\begin{array}{l}30 \text { Vídeos } \\
\text { Lanz. Juego }\end{array}$ & \\
\hline VJ1. Zona de comienzo & 0.72 & SUS & 0.84 & C.P. & 0.91 & \\
\hline VJ2. Tipo de progreso & 0.84 & C.P. & 0.87 & C.P. & 0.91 & \\
\hline VJ3. Zona de lanzamiento & 0.99 & C.P. & 0.84 & C.P. & 0.97 & \\
\hline VJ4. Situación de golpeo & 0.99 & C.P. & 1.00 & C.P. & 0.97 & \\
\hline VJ5. Bloqueos & 0.99 & C.P. & 0.94 & C.P. & 0.98 & \\
\hline VJ6. Oposición lanzamiento & 0.86 & C.P. & 0.81 & C.P. & 0.92 & \\
\hline VJ7. Zona corporal & 0.96 & C.P. & 1.00 & C.P. & 1.00 & \\
\hline VJ8. Tipo de golpeo & 0.81 & C.P. & 0.71 & sUS & 0.86 & \\
\hline VJ8*. Tipo de golpeo & 0.86 & C.P. & 0.89 & C.P. & 0.87 & \\
\hline VJ9. Resultado en juego & 0.89 & C.P. & 0.89 & C.P. & 0.93 & \\
\hline
\end{tabular}

E. A. Etiquetas de Acuerdo según Landis \& Koch (1977).

P - Pobre; L - Leve; J - Justo; M - Moderado; SUS - Sustancial; C.P. - Casi Perfecto. Lanz. Lanzamientos; * variables recodificadas.

Tabla 5.

\begin{tabular}{llll} 
Promedio de los datos recogidos por los codificadores. & \\
\hline 10 & 20 & 30
\end{tabular}

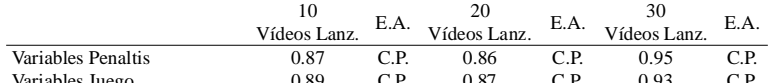
Variables Juego Total

Variables Penaltis

Variables Juego *

Total *

$0.90 \quad$ C.P.

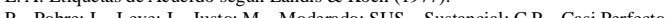
Lanz. Lanzamientos; * con variables recodificadas.

\section{Resultados}

En la Tabla 4 se muestra la evolución del grado de acuerdo en los ejercicios de la fase de entrenamiento, concretamente para las acciones básicas durante el lanzamiento de penalti y las acciones básicas durante el lanzamiento a portería en situaciones de juego. Esta fase se planifica con un aumento progresivo en el número de acciones a analizar hasta llegar a un partido de juego completo de un partido, que es similar a las unidades de observación que se desarrollan para la codificación de la muestra.

La mayoría de las variables presenta un nivel de acuerdo alto desde el inicio del proceso de formación de codificadores. Tan sólo la VP4, Tipo de golpeo, no llega a obtener un valor de Kappa superior a 0.70, lo que indica el nivel de acuerdo es bajo para el valor de referencia establecido en este estudio, a pesar de ser «sustancial» (Landis \& Koch, 1977). Por tanto, con el objetivo de alcanzar un mayor nivel concordancia, y teniendo en cuenta el origen de los errores entre codificadores planteado por James, Taylor \& Stanley (2007) y Anguera (1988), se desarrollo un proceso de recodificación de la variable VP4 y VJ8 (Figura 2).

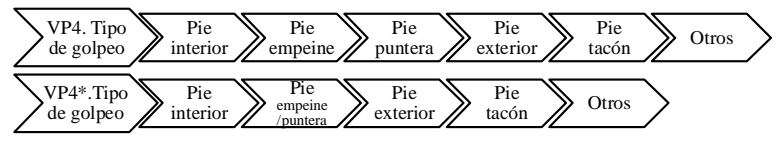

Figura 2. Esquema de recodificación de variable.

En la tabla 5 se muestra la evolución de la fiabilidad de los datos recogidos por los codificadores durante la fase práctica de observación y fase de entrenamiento individual. Durante el proceso de formación se aprecia una mejora parcial y total de concordancia entre los codificadores en cada una de las variables del estudio.

\section{Discusión}

En este estudio se ha desarrollado y evaluado un proceso de formación de codificadores para el análisis del rendimiento competitivo en Fa5. Es decir, se ha descrito un proceso de formación y evaluación de la fiabilidad inter-observador de los codificadores participantes. Para ello, es necesario analizar la fiabilidad obtenida entre los diferentes codificadores sometidos a un proceso de formación teórica. La formación de observa- dores desarrollada en el presente estudio cumple con las premisas planteadas por Medina \& Delgado (1999) así como en estudios previos de esta naturaleza (Sarmento et al., 2010). Para facilitar la compresión del proceso de formación se ha dividido en cuatro etapas: etapa preparatoria; etapa de selección de los codificadores; etapa de formación de los observadores; y por último, etapa de confiabilidad.

1) Etapa preparatoria. Es una etapa clave en cualquier estudio de formación de codificadores. La metodología observacional supone seguir un proceso de formación disciplinada y rigurosa. Por esto, se debe de seleccionar y preparar los documentos, definir los objetivos e hipótesis y determinar la unidad de registro a emplear (Moreno et al., 2002) de forma metódica y antes de empezar el proceso. Se evitarán fallos que puedan surgir durante el proceso de formación y se estará asegurando que la recogida de los datos sea objetiva (Anguera et al., 1999; Losada \& Manolov, 2014; Medina \& Delgado, 1999). Además, en esta etapa se debe de planificar y elaborar los materiales e instrumentos (Viciana, 1999) que utilizaran los codificadores para obtener datos fiables sobre el fútbol a 5 para personas ciegas. La escasez de referencias concretas a las etapas y fases que constituyen el proceso de formación de observadores, ha llevado a los investigadores a exponer de forma detalla cada una de las partes que constituye la investigación. Los trabajos que adaptan la propuesta de formación de codificadores de Medina \& Delgado (1999), detallando las diferentes etapas o fases seguidas en el proceso (Arias et al., 2009; Viciana, 1999; Moreno et al., 2002) no tienen en cuenta la etapa previa o proponen otras como en los trabajos deAnguera et al. (1999) o Losada \& Manolov (2014). A pesar de su importancia en el adiestramiento y formación de observadores según indica Anguera (1991).

Las diferentes etapas del proceso de formación están planificadas con la intención de iniciar al codificador en el conocimiento del fútbol a 5 para personas ciegas, recogida de datos, como paso previo a la obtención de fiabilidad en la codificación. Arias et al., (2009) dividen esta etapa en dos fases: elaboración del instrumento de observación y del instrumento de registro; y la otra, es elaboración del manual de formación y del manual de instrucciones para los observadores. Por esto, se debe agrupar en esta etapa todo lo que conlleva la preparación del proceso de formación de codificadores: objetivos del estudio, manuales, instrumentos a utilizar, sistema y medios de recogida de datos, etc. Además, se debe elaborar una lista de comportamientos a partir de la literatura científica y la consulta de expertos (Villarejo et al., 2014). Por tanto, esta etapa es clave para que todas las demás etapas sean efectivas, permitiendo dar respuesta a los objetivos diversos que propagan los investigadores en sus estudios y adecuándose de forma óptima a las especificidades que interesen en función de la modalidad deportiva de estudio (Anguera \& Hernández-Mendo, 2015).

2) Etapa de Selección de los codificadores. En la literatura existente el número de codificadores que intervienen durante el proceso de formación varía según los autores. Seleccionar un grupo de codificadores es importante para que el procedimiento de recogida sea un éxito. Nadeau et al., (2008) proponen el empleo de dos estudiantes-observadores asignados para analizar cada jugador de hockey durante el juego son bastantes para obtener fiabilidad. Igualmente, Taylor et al., (2009) proponen dos observadores experimentados para registrar los datos de fútbol. Morato et al., (2016) seleccionan dos observadores expertos para el estudio de indicadores en goalball.

Además, hay trabajos con tres codificadores capacitados en el uso del instrumento de observación (Blanco, Ibáñez, Antúnez \& HernándezMendo, 2015; Santos et al., 2014; Villarejo et al.,2014). Otros estudios proponen cuatro codificadores para desarrollar el proceso de formación así como para la recogida de datos (Alonso \& Argudo, 2008; Arias et al., 2009; Casal et al., 2015). En el estudio de Hernández-Mendo et al. (2012) participaron seis personas, divididas en dos grupos para realizar observaciones no planificadas y sistemáticas. Catalán-Eslava \& González (2015) seleccionan seis codificadores para el proceso de formación así como para registrar los datos. Serra-Olivares \& García- 
López (2016) plantean siete codificadores para diseñar y validar el instrumento TCTOF. Medina (1996) selecciona ocho sujetos a entrenar. Otros estudios cuentan con diez codificadores sin requisitos de participación (Moreno et al., 2002). Sin embargo, en el trabajo de Robles-Rodríguez, Robles-Rodríguez, Giménez Fuentes-Guerra \& Abad (2016) participaron diez codificadores expertos, todos con la titulación de doctor en ciencias de la actividad física y del deporte. Otros autores proponen once codificadores (García-Martín, Antúnez \& Ibáñez, 2016; Sousa, Prudente, Sequeira \& Hernández-Mendo, 2013) o con quince observadores divididos en tres grupos de cinco (Viciana, 1999). Por último, el estudio de Usabiaga et al. (2013) incluyó a ocho pares de observadores para garantizar la concordancia consensuada de Anguera (1991). Por último, hay trabajos que no citan el número de codificadores a pesar de utilizar un protocolo de observación y un entrenamiento minucioso de los observadores (Ibáñez et al., 2009; Morillo \& Hernández-Mendo, 2015).

En este estudio, se propuso cinco codificadores seleccionados que cumplen con los criterios de inclusión establecidos. Número de codificadores suficiente para asegurar la correcta recogida de datos. Todos los sujetos eran capaces de transmitir conocimientos e informaciones acerca del objeto de estudio. Los criterios de selección del estudio muestran similitudes con respecto a los de otros trabajos (Medina, 1996 o García-Martín et al., 2016), es decir, se seleccionaron profesionales de la Actividad Física y el Deporte y la Educación Física, con formación inicial y conocimientos suficientes sobre el tema de estudio debido a la dificultad por la especificad del contenido. Además, implicación en el proceso y experiencia en ese ámbito (Moreno et al., 2002). Otro criterio, experiencia como investigador en el área del deporte adaptado cumpliendo así con uno de los ítems de inclusión del estudio de Santos et al., (2014). Por tanto, seleccionar un grupo de codificadores óptimos es clave para que el procedimiento de recogida sea un éxito, así como que sean los más adecuados para el estudio.

3) Etapa de Formación de los codificadores. Esta etapa se dividió en tres fases que debían de seguir los investigadores y los codificadores durante el proceso de formación: fase de formación teórica, fase de formación práctica y fase de entrenamiento individual. Por tanto, se involucró a los codificadores durante su proceso de formación para la recodificación de las categorías a observar, con el fin de mejorar de forma constructiva algunas de las propuestas de formación de entrenadores existentes en la literatura (Arias et al., 2009; Moreno et al., 2002; Villarejo et al., 2014).

3.1) Fase de formación teórica de los observadores. En esta fase se desarrollan una serie de objetivos: conocer la conducta a observar, realizar ejercicio práctico de observación, hacer reflexionar al grupo de observadores sobre determinadas conductas del estudio, conocer las categorías existentes, facilitar la compresión, resolver dudas a los observadores, comprender y corregir los ejemplos para facilitar una mayor compresión de las categorías a registrar (Medina \& Delgado, 1999; Losada \& Manolov (2014). Por tanto, tendrá especial importancia la concreción de sesiones planteas para el proceso de investigación. En la literatura existente, hay autores que citan una cantidad de sesiones (Arias et al., 2009; Morato et al., 2016; Moreno et al., 2002; Villarejo et al., 2014) uhoras (Lago, 2009; Taylor et al., 2009; Usabiaga et al., 2013) necesarias para plantear el proceso de formación presencial. Por último, hay trabajos que no citan el número de sesiones o el total horas empleadas en la fase de formación teórica de los observadores (Alonso et al., 2008; Casal et al., 2015; Ibáñez et al., 2009; Morillo et al., 2015; Nadeau et al., 2008; Santos et al., 2014) a pesar de utilizar un proceso de entrenamiento para observadores minuciosos (Morillo \& HernándezMendo, 2015). Sin embargo, Viciana (1999) expone que el número de sesiones será variable en función de los contenidos a programar.

En el presente trabajo, se opta por un número de siete sesiones condicionado por los contenidos del estudio. El total entre todas las sesiones fue de cinco horas y media. Número de horas suficientes para desarrollar los objetivos propuestos por Medina \& Delgado (1999).
Son sesiones de exploración que permiten delimitar el problema de investigación y reducir la ocurrencia de errores en el futuro (Anguera \& Hernández-Mendo, 2015).

3.2) Fase de formación práctica de los observadores. Duranteel desarrollo de la investigación se debe comprobar con determinada frecuencia la fiabilidad de los observadores, así se asegurará que los registros están cumpliendo con los requisitos mínimos de fiabilidad (objetividad) exigida (Medina \& Delgado, 1999) y se debe desarrollar hasta conseguir una adecuada fiabilidad intercodificadores (Moreno et al., 2002). A partir de entonces los codificadores estarán preparados para codificar individualmente, garantizando la fiabilidad (Medina, 1996; Viciana, 1999). La fase de formación práctica se diseñó siguiendo un criterio básico de simplicidad, basado en ejercicios individuales. Estas sesiones de entrenamiento se programan de manera progresiva, tal como plantea Medina (1996). Por tanto, en esta fase se inicia la evaluación del nivel de acuerdo inter-codificador, que posteriormente se desarrollará para estudiar la fiabilidad y la validez.

El primer ejercicio de la fase de formación práctica de los observadores, se contaba con diez lanzamientos de lanzamiento en juego y de penalti. Se aprecia que la mayoría de las variables tienen un alto grado de acuerdo (>0.70) (Randolph, 2005). Esto significa que la fase de formación teórica ha sido positiva. Los codificadores han entendido las variables a registrar. Además, tras la conclusión del ejercicio se facilitó a los observadores un informe de resultados y recomendaciones para seguir mejorando los registros. Este proceso se planteó también en la fase de entrenamiento individual.

3.3) Fase de entrenamiento individual. En esta fase los ejercicios de observación se vuelven a realizar de forma individual, evaluando la fiabilidad de cada uno. El segundo ejercicio de la fase de entrenamiento individual, que contaba con veinte lanzamientos en juego y de penalti. Se aprecia que la totalidad de las variables tienen un alto grado de acuerdo (Randolph, 2005), a excepción de la variable VP4. Esto significa la existencia de dudas o errores por parte de los observadores. Por tanto, las fuentes de error a las que se ven sometidos los codificadores pueden resolverse mediante una buena planificación y adecuado adiestramiento de los mismos (Anguera, 1988). Se optó por recordar a los codificadores los principales aspectos a tener en cuenta (Viciana, 1999) para evitar posibles errores en las codificaciones. Se realizó el procedimiento de recodificación de variables, concretamente de las variables VP4 y VJ8. De esta manera, se evita algunos de los errores que provocan la falta de acuerdo entre los observadores y por tanto una baja fiabilidad inter-observadores. Los errores por una baja fiabilidad pueden ser: Error Operacional, Error Observacional y/o Error de Definición (James et al., 2007). En este caso, eran del tercer tipo, un Error de Definición. Dos de las categorías de esta variable eran muy similares y por tanto difíciles de diferenciar en las imágenes por el ángulo de grabación de la cámara. De está forma las variables VP4 y VJ8 se recodificaron de seis categorías a cinco, uniendo las categorías dos y tres, además de dar una nueva numeración que ordena las mismas de forma más lógica. La VJ8 se recodificó para evitar posibles errores pues son iguales en ambas acciones del estudio.

Además, citar que las tres fases que debían de seguir los investigadores y los codificadores durante el proceso de formación: fase de formación teórica, fase de formación práctica y fase de entrenamiento individual durarán dependiendo de la dificultad por la especificidad del estudio. Arias et al., (2009) citan que el proceso de formación de los codificadores duró dos semanas. En el estudio de Alonso \& Argudo (2008) el proceso de entrenamiento de los observadores se desarrolló en cuatro semanas. Viciana, (1999) planificó una serie de seminario específicos de cincuenta horas, más una serie de tareas para realizar en horario extra. En este estudio, se desarrolló durante doce semanas debido a la complejidad del estudio y disponibilidad de los codificadores.

4) Etapa de confiabilidad. Por último, la etapa de confiabilidad tiene la finalidad de comprobar y garantizar que la muestra del estudio 
tiene un alto grado de objetividad, y por tanto el rigor necesario para la investigación. Durante el proceso de formación de codificadores en Fa5 se aprecia una mejora parcial y total de la concordancia entre los codificadores. Muestran un valor de Kappa de 0.95, superior al de otros trabajos de investigación (Den Hollander, Brown, Lambert, Treu \& Hendricks, 2016; Cañadas, Ibáñez \& Leite, 2015; Ibáñez, Santos \& García, 2015), e igual a otros estudios de fútbol (Sarmento et al., 2010). La confiabilidad se estableció utilizando el acuerdo entre los codificadores de forma parcial y total. Por tanto, se puede afirmar que la fiabilidad del procedimiento es casi perfecto (Landis \& Koch, 1977), y sus datos son superiores a otras investigaciones previas.

\section{Conclusiones}

El estudio de entrenamiento y confiabilidad entre observadores para el análisis del Fa5 tiene por finalidad describir el proceso de formación y por tanto, confirmar si los datos son validos y fiables pondrán ser empleados para la investigación. Se propone un diseño y entrenamiento de los codificadores dividido en cuatro etapas: etapa preparatoria; etapa de selección de los codificadores; etapa de formación de los observadores; y por último, etapa de confiabilidad.

La etapa preparatoria es clave para tener éxito en las restantes fases del proceso de entrenamiento. El diseño, y organización de los archivos que manipularán los codificadores deben estar identificados y numerados con claridad para facilitar su localización en las distintas herramientas que se emplearán y utilizarán para la recogida de datos. Así como que todos los recursos estén disponibles permanentemente para los observadores.

Los observadores seleccionados en este estudio tienen que cumplir con los criterios de inclusión establecidos. Poseen una alta calidad para asegurar un alto acuerdo en todas las variables a analizar.

La etapa de formación de los observadores se dividió en tres fases: fase de formación teórica, fase de formación práctica y fase de entrenamiento individual. Cada fase tiene una finalidad concreta. La primera fase permite conocer el objeto de investigación y reducir las dudas y errores durante el proceso de entrenamiento. La siguiente fase permite determinar la frecuencia de la fiabilidad entre los observadores. Y la fase de entrenamiento individual se realiza de forma individual, evaluando la fiabilidad de cada observador de manera particular.

La duración de la etapa de confiabilidad estará en función del grado de acuerdo que se alcance. Esta etapa determinará si el procedimiento para el análisis del Fa5 es válido. Por tanto, el procedimiento de entrenamiento y confiabilidad presentado en este trabajo puede ser usado en el ámbito de las Ciencias de la Actividad Física y el Deporte, y concretamente en el contexto del Fa5.

\section{Referencias}

Alonso, J. L., \& Argudo, F. (2008). Indicadores de rendimiento del saque en frontenis olímpico femenino. Revista Internacional de Ciencias del Deporte, 10, 59-76.

Anguera, M. T. (1988). Observación en la escuela. Barcelona: Graó.

Anguera, M. T. (1991). Metodología observacional en la investigación psicológica. Barcelona: PPU.

Anguera, M. T., \& Hernández-Mendo, A. (2013). La metodología observacional en el ámbito del deporte. E-Balonmano: Revista de Ciencias del Deporte, 9(3), 135-160.

Anguera, M. T., \& Hernández-Mendo, A. (2014). Metodología observacional y psicología del deporte: Estado de la cuestión. Revista de Psicología del Deporte, 23(1), 103-109.

Anguera, M. T., \& Hernández-Mendo, A. (2015). Técnicas de análisis en estudios observacionales en ciencias del deporte. Cuadernos de Psicología del Deporte, 15(1), 13-30.

Anguera, M. T., Blanco, A., Losada, J.L. y Sánchez-Algarra, P. (1999). Análisis de la competencia en la selección de observadores. Metodología de las Ciencias del Comportamiento, 1(1), 95-114.

Arias, J., Argudo, F., \& Alonso, J. (2009). El proceso de formación de observadores y la obtención de la fiabilidad en metodología observacional para analizar la dinámica de juego en minibásquet. Apunts: Educación Física y Deporte, 98 (4), 40-45.

Barraza, A. (2007). Apuntes sobre metodología de la investigación: confiabilidad. Revista INED, 2(6), 6-10.

Blanco, A., Ibáñez, S. J.,Antúnez, A., \& Hernández-Mendo,A. (2015). Estudio de fiabilidad de los indicadores de rendimiento en Liga Asobal. Cuadernos de Psicología del Deporte, 15(1), 255-264.

Brennan, R. L., \& Prediger, D. J. (1981). Coefficient kappa-some uses, misuses, and alternatives. Educational and Psychological Measurement, 41(3), 687-699.

Cañadas, M., Ibáñez, S. J., \& Leite, N. (2015).Anovice coach's planning of the technical and tactical content of youth basketball training: a case study. International Journal of Performance Analysis in Sport, 15, 572-587.

Caro, O., \& Caro-Muñoz, A. (2016). Aproximación a los modelos tácticos generales ofensivos mediantes el análisis de los goles en fútbol profesional. Journal of Sport and Health Research, 8(1), 112.

Casal, C., Losada, J.L., \& Ardá, T. (2015). Análisis de los factores de rendimiento de las transiciones ofensivas en el fútbol de alto nivel. Revistas de Psicología del Deporte, 24(1), 103-110.

Castellano, J., \& Hernández-Mendo,A. (2015). La observación aplicada en diferentes ámbitos de la actividad física y el deporte: los fundamentos de base. Revista Española de Educción Física y Deportes, (409), 15-19.

Catalán-Eslava, M., \& González- Villora, S. (2015). Validación de un instrumento de evaluación en deportes dered-muro: squash(HERS). RETOS. Nuevas Tendencias en Educación Física, Deporte y Recreación, 27, 73-80.

Den Hollander, S., Brown, J., Lambert, M., Treu, P., \& Hendricks, S. (2016). Skills Associated with Line Breaks in Elite Rugby Union. Journal of Sport Science \& Medicine, 15(3), 501-508.

Drust, B. (2010). Performance analysis research: Meeting the challenge. Journal of Sport Sciences, 28(9), 921-922.

Escobar J., \& Cuervo, A. (2008). Validez de contenido y juicio de expertos: una aproximación a su utilización. Avances en Medición, 6, 27-36.

Fleiss, J. L., Levin, B., \& Paik, M. C. (2013). Statistal methods for rates and proportions. $3^{\circ}$ ed. New Jersey: John Wiley \& Sons.

Gamonales, J. M. (2016). Fútbol para personas ciegas y con deficiencia visual: «Un mundo sin descubrir». En Gallardo, D., \& Urraco, M. (2016). Catálogo de Investigación joven en Extremadura. Edición 2015 (cap. 29). Cáceres: Servicio de Publicaciones de la Universidad de Extremadura.

Gamonales, J. M. (2017). Fútbol a 5 para personas ciegas como contenido de Educación Física. Revista Profesional de Docenciay Recursos Didácticos, 80(3), 66-70.

Gamonales, J. M., León, K., Muñoz, J., González-Espinosa, S., \& Ibáñez, S. J. (In press). Validación del IOLF5C para la eficacia del lanzamiento en fútbol para ciegos. Revista Internacional de Medicinay Ciencias del Deporte, $x(\mathrm{x}), \mathrm{xx}-\mathrm{xx}$. Pendiente de publicación / In press.

García-Martín, A., Antúnez, A., \& Ibañez, S.J. (2016). Análisis del proceso formativo en jugadores expertos: validación de instrumento. Revista Internacional de Medicina y Ciencias de la Actividad Física y el Deporte, 16(61), 157-182.

Gómez-Ruano, M. (2017). La importancia del análisis notacional como tópico emergente en Ciencias del Deporte. RICYDE, Revista Internacional de Ciencias del Deporte, 13(47), 1-4.

Hernández-Mendo, A., Montoro, J., Reina, A., \& Fernández, J. C. (2012). Desarrollo y optimización de una herramienta observacional para el bloqueo en voleibol. Revista Iberoamericana de Psicología del Ejercicio y el Deporte, 7(1), 15-31.

Hernández, J. (2005). Análisis de las estructuras del juego deportivo (3rd ed.). Barcelona: INDE.

Hughes, M. D., \& Bartlett, R. M. (2002). The use of performance 
indicators in performance analysis. Journal of Sports Sciences, 20(10), 739-754.

Ibáñez, S. J., García, J., Feu, S., Parejo, I., \& Cañadas, M. (2009). La eficacia del lanzamiento a canasta en la NBA:Análisis multifactorial. Cultura, Ciencia y Deporte, 4(10), 39-47.

Ibáñez, S. J., Santos, J. A., \& García, J. (2015). Multifactorial analysis or free throw shooting in eliminatory basketball games. International Journal of Performance Analysis in Sport, 15, 897-912.

IBSA-International Blind Sports Federation (2017). Fútbol - Información general. Consultado en http://www.ibsasport.org/sports/ football/rules/

James, N., Taylor, J. B., \& Stanley, S. (2007). Reliability procedures for categorical data in performance analysis. International Journal of Performance Analysis in Sport, 7, 1-11.

Lago, C. (2009). The influence of match location, quality of oppostion, and mach status on possession strategies in profesional association football. Journal of Sport Sciences, 27(13), 130-137.

Landis, J. R. \& Koch, G. G. (1977). Aplication of hierarchical kappatype statistics in assessment of majority agreement among multiple observers. Biometrics, 33(2), 363-374.

Losada, J. L. \& Manolov, R. (2015). The process of basic training, applied

training, maintaining the performance of an observer. Quality \& Quantity, 49(1), 339-347.

Losada, J. L., \& Manolov, R. (2014). The process of basic training, applied training, maintaining the performance of an observer. Quality \& Quantity, 49(1), 339-347.

Matsui, R. (2017). I Jogoa Escolares Brasileiros da Confederação Brasileira de Desportos para Cegos: Um estudo de caso. Programa de Graduados en Educación Física. Universidad Estatal de Campinas. Facultad de Educación Física. Campinas (Brasil).

McGarry, T. (2009). Applied and theoretical perspectives of performance analysis in sport: Scientific issues and challenges. International Journal of Performance Analysis in Sport, 9(1), 128-140.

Medina, J. (1996). Proceso de entrenamiento de codificadores para el estudio de los diarios del profesorado de educación física. Revista Motricidad, 2, 113-127.

Medina, J., \& Delgado, M.A. (1999) Metodología de entrenamiento de observadores para investigaciones sobre Educación Física y Deporte en las que se utilice como método la observación. Revista Motricidad, 5, 69-86.

Montero, I., G., \& León O. (2007). A guide for naming research studies in Phychology. International Journal of Clinical and Health Psychology, 7(3), 847-862.

Morato, M., Bilzon, J., \& Duarte, E. (2013). Sports injuries in Brazilian blind footballers. Revista Movimento, 17(3), 97-114.

Morato, M., Da Cunha, O., Gamero, D., Magalhães, T., \& Almeida, J. (2016). Development and evaluation of an observational system for goalball match analysis. Revista Brasileira de Ciências do Esporte, xx(xx), 1-10. http://dx.doi.org/10.1016/j.rbce.08.002

Moreno, M. P., Santos, J. A., Ramos, L. A., Sanz, D., Fuentes, J. P., \& Del Villar, F. (2002). Aplicación de un sistema de codificación para el análisis de contenido de la conducta verbal del entrenador de voleibol. European Journal of Human Movement, (9), 119-140.
Morillo, J., \& Hernández-Mendo, A. (2015). Análisis de la Calidad del Dato de un instrumento para la observación del ataque en balonmano playa. Revista Iberoamérica de Psicología del Ejercicio yel Deporte, 10(1), 15-22.

Nadeau, L., Richard, J. F., \& Godbout, P. (2008). The validity and reliability of a performance assessment procedure in ice hockey. Physical Education and Sport Pedagogy, 13(1), 65-83.

Nevill, A. M., Atkinson, G., \& Hughes, M. D. (2008) Twenty-five years of sport performance research in the journal of sport sciences. Journal of Sports Sciences, 26(4), 413-426.

Randolph, J. J. (2005). Free-Marginal Multirater Kappa (multirater Kfree): AnAlternative to Fleiss' Fixed-Marginal Multirater Kappa. Joensuu Learning and Instruction Symposium 2005, University of the Joensuu, Finland.

Robles-Rodríguez, A., Robles-Rodríguez, J., Giménez Fuentes-Guerra, F.J., \& Abad Robles, M.T. (2016). Validación de una entrevista para estudiar el proceso formativo de judokas de élite. Revista Internacional de Medicina y Ciencias de la Actividad Física y el Deporte, 16(64), 723-738.

Sampaio, J., Ibáñez, S. J., \& Lorenzo, A. (2013). Basketball. In McGarry T., O’Donoghue, P., Sampaio, J., Eds. Routledg handbook of Sports performance analysis. (357-366). Routledge: London.

Santos, S., Sarmento, H., Alves, J., \& Campanico, J. (2014). Construcción de un instrumento para la observación y el análisis de las interacciones en el waterpolo. Revista de Psicología del Deporte, 23(1), 191-200.

Sarmento, H., Anguera, M. T., Campaniço, J., \& Leitão, J. (2010). Development and validation of a notacional system to study the offensive process in football. Medicina (Kaunas), 46(6), 401-407.

Serra-Olivares, J., \& García-López, L.M. (2016). Diseño y validación del test de conocimiento táctico ofensivo en fútbol (TCTOF). Revista Internacional de Medicina y Ciencias de la Actividad Física y el Deporte, 16(62), 521-536.

Sousa, D., Prudente, J., Sequeira, P., \& Hernández-Mendo, A. (2013). Análise da qualidade dos dados de um instrumento para observaçao do 2 vs 2 no andebol. Revista Iberoamericana de Psicología del Ejercicio y el Deporte, 9(1), 173-190.

Taylor, J. B., Mellalieu, S. O., James, N., \& Shearer, D. A (2009). The influence of match location, quality of oppsition, and match status on technical performance in profesional association football. Journal of Sport Sciences, 26(9), 885-895.

Usabiaga, O., Castellano, J., Blanco-Villaseñor,A., \& Casamichana, D. (2013). La Teoría de la Generalizabilidad en las primeras fases del método observacional aplicado en el ámbito de la iniciación deportiva: calidad del dato y estimación de la muestra. Revista de Psicología del Deporte, 22(1), 103-109.

Viciana, J.(1999). Proceso de entrenamiento de grupos de codificadores específicos antes un sistema múltiple de categorías de análisis del discurso en un grupo de trabajo de docentes en educación física. Revista Motricidad, 5(1), 53-67.

Villarejo, D., Ortega, E., Gómez, M. A., \& Palao, J. (2014). Design, validation and reliability of an observational instrument for ball possessions in rugby unión. International Journal of Performance Analysis, 14, 896-908.

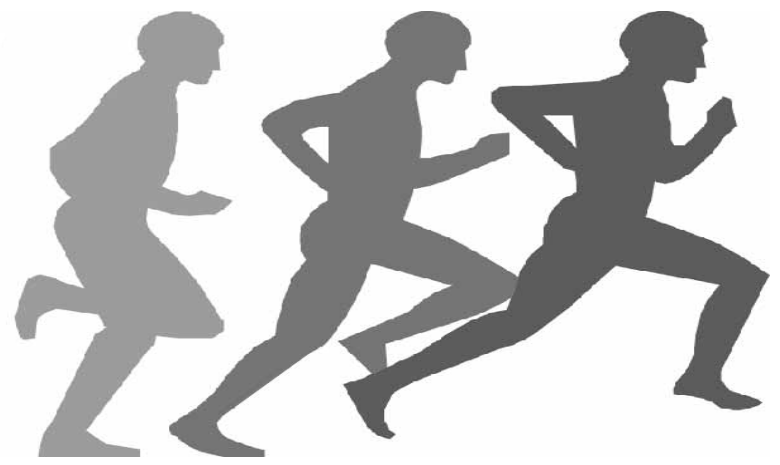

